\title{
Hybrid Golay-coded Brillouin optical time-domain analysis based on differential pulses
}

\author{
Zonglei Li, ${ }^{1,2}$ (i) Zhisheng Yang, ${ }^{1, *}$ (i) Lianshan Yan, ${ }^{2}$ (i) Marcelo A. Soto, ${ }^{1,3}$ (i) and Luc Thévenaz ${ }^{1}$ (i) \\ ${ }^{1}$ EPFL Swiss Federal Institute of Technology, Institute of Electrical Engineering, SCI STI LT, Station 11, CH-1015 Lausanne, Switzerland \\ ${ }^{2}$ Center for Information Photonics \& Communications, School of Information Science \& Technology, Southwest Jiaotong University, \\ Cheng du, Sichuan 610031, China \\ ${ }^{3}$ Department of Electronic Engineering, Universidad Técnica Federico Santa María, 2390123 Valparaíso, Chile \\ *Corresponding author: zhisheng.yang@epfl.ch
}

Received 25 June 2018; revised 21 August 2018; accepted 22 August 2018; posted 23 August 2018 (Doc. ID 335882); published 17 September 2018

\begin{abstract}
Different approaches to implement unipolar Golay coding in Brillouin optical time-domain analysis based on a differential pulse pair (DPP) are investigated. The analysis points out that dedicated post-processing procedures must be followed to secure the sharp spatial resolution associated with the DPP method. Moreover, a novel hybrid Golay-DPP coding scheme is proposed, offering $1.5 \mathrm{~dB}$ signal-to-noise ratio improvement with respect to traditional unipolar Golay coding, while halving the measurement time, constituting a $3 \mathrm{~dB}$ overall coding gain enhancement. Proof-of-concept experiments validate the proposed technique, demonstrating a $50 \mathrm{~cm}$ spatial resolution over a $10.164 \mathrm{~km}$ long sensing fiber with a frequency uncertainty of $1.4 \mathrm{MHz}$. () 2018 Optical Society of America
\end{abstract}

OCIS codes: (060.2370) Fiber optics sensors; (290.5900) Scattering, stimulated Brillouin.

https://doi.org/10.1364/OL.43.004574

Provided under the terms of the OSA Open Access Publishing Agreement

Brillouin optical time-domain analysis (BOTDA) has been studied for more than two decades, since it can provide reliable and long-range distributed temperature and strain sensing [1,2]. After active years of research in this field, today's mature theory points out that the overall performance of BOTDA systems is ultimately scaled by the signal-to-noise ratio (SNR) of the measurements, the pump-probe frequency offset scanning step, and the full-width at half-maximum (FWHM) of the measured Brillouin gain spectrum (BGS) [2]. In classical BOTDA schemes, achieving sharp (sub-meter) spatial resolution turns challenging, since the use of a short optical pulse results in a spectrally broadened BGS and a significant reduction of the SBS peak gain [3]. To avoid those detrimental effects, several interrogation techniques have been proposed in the literature. Among them, the concept of differential pulse pair (DPP) $[4,5]$ turns out to be efficient to obtain sharp spatial resolutions without BGS degradation, while requiring one additional acquisition process with respect to a standard BOTDA. Since this technique is essentially similar to the conventional single-pulse BOTDA, the SNR of the measured traces is also limited by nonlinear effects taking place in the sensing fiber [6,7]. Since optical pulse coding can increase the SNR by factor $\sqrt{M} / 2$ ( $M$ is the code length) [8-10], while keeping the same pulse peak power and similar measurement time, Simplex coding has been well demonstrated in DPP-BOTDA systems to improve the SNR [11], in which the decoded traces associated with the long and short pulses used in DPP are simply subtracted.

Considering that Golay codes can provide the same coding gain as Simplex codes with less data storage and shorter postprocessing time, in this Letter, we investigate the viability of Golay coding implementation in DPP-BOTDA sensing. It is found out that, in contrast to the Simplex coding approach, the coding/decoding process followed in Golay-coded DPPBOTDA systems might easily lead to worse spatial resolutions, which do not match the value expected from the differential pulse. To overcome this detrimental behavior, a novel hybrid coding method is proposed and experimentally validated. The technique halves the measurement time and increases the measurement SNR by $1.5 \mathrm{~dB}$ over classical unipolar Golay codes, constituting a $3 \mathrm{~dB}$ overall coding gain improvement on a basis of similar acquisition times.

The decoding of Golay codes relies on the autocorrelation properties of the complementary code sequences. In an ideal case, the sum of the autocorrelation functions of Golay code sequences, whose amplitude is then normalized by twice that of the product between the coding length and the number of sampled points within the pulse width, denoted as interrogation function, possesses a main correlation peak and no sidelobes $[10,12]$. This main correlation peak interrogates the local Brillouin gain along the fiber, in total similarity with the behavior of the single pump pulse in standard BOTDA. Considering that each code element, i.e., each optical pulse, has a square temporal profile, the main correlation peak of the interrogation function exhibits a triangle whose FWHM is equal to the pulse width, so that the spatial resolution of the sensor is maintained after decoding [12]. Here we point out that for some specific cases, e.g., when combining a unipolar Golay coding technique with the DPP method, this triangular temporal profile can impair the spatial resolution if decoding is not suitably performed.

Similar to Simplex-coded DPP-BOTDA $[8,11]$, the most straightforward way to implement a unipolar Golay-coded 
DPP-BOTDA is to first carry out measurements and a decoding procedure for the sequences with long pulses [width $=T_{L}$ in Fig. 1(a)] and then do the same for the sequences with short pulses [width $=T_{S}$ in Fig. 1(b)], both processes being independent. By subtracting these two decoded responses, a sharp spatial resolution determined by $T_{L}-T_{S}$ is expected to be obtained. Since the process initially performs cross-correlation to decode the traces, and then conducts a subtraction operation to obtain the differential gain, this approach is denominated the "C-S method" in this Letter. As previously described, the interrogation functions for the long and short pulses $\left(f_{L}\right.$ and $\left.f_{S}\right)$ exhibit triangular shapes with the same amplitude, but different FWHMs, as shown in Fig. 2, in magenta and blue colors, respectively, for a 30-25 ns DPP-BOTDA system. After subtraction, the resulted interrogating function is $f_{C-S}^{\text {DPP }}=f_{L}-f_{S}$, as shown by the brown line in Fig. 2. This $f_{C-S}^{\text {DPP }}$ function, besides being distorted, carries the information over the entire fiber section covered by the long pulse, resulting in a spatial resolution equal to the one determined by the long pulse $T_{L}$ instead of the expected differential pulse width $T_{L}-T_{S}$ (i.e., the system represented in Fig. 2 results in a $3 \mathrm{~m}$ resolution instead of the expected $50 \mathrm{~cm}$ ).

Actually, this degradation of spatial resolution using the C-S method can be readily overcome by re-ordering the post-processing procedure, namely the S-C method, by first subtracting the traces

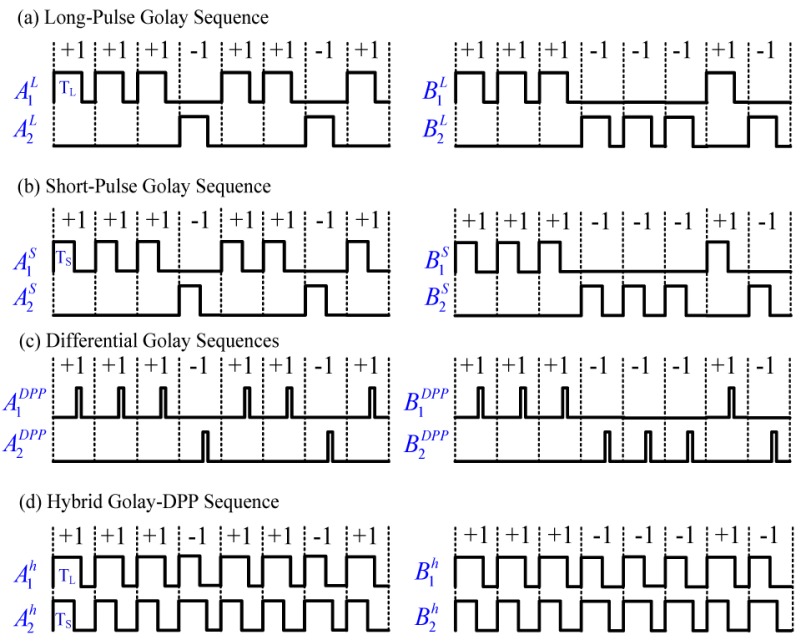

Fig. 1. Temporal shapes of (a) long-pulse, (b) short-pulse, (c) differential-pulse, and (d) hybrid-pulse Golay code sequences.

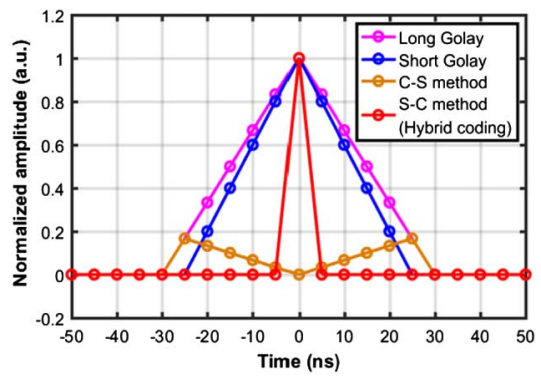

Fig. 2. Simulated Golay coding interrogating functions $f_{L}$ (longpulse Golay sequences), $f_{S}$ (short-pulse Golay sequences), $f_{C-S}^{\mathrm{DPP}}$ (C-S method), and $f_{S-C}^{\text {DPP }}$ (S-C method). coded under different pulse widths, and then decoding by crosscorrelating the coded DPP traces. Defining $R A_{n}^{L}, R A_{n}^{S}, R B_{n}^{L}$, and $R B_{n}^{S}$ as the BOTDA responses associated with each code sequence $\left(A_{n}^{L}, A_{n}^{S}, B_{n}^{L}, B_{n}^{S}\right)$, the corresponding differential Brillouin responses $R A_{n}^{\mathrm{DPP}}$ and $R B_{n}^{\mathrm{DPP}}$ for the $\mathrm{S}-\mathrm{C}$ method can be written as

$$
\left\{\begin{array}{l}
R A_{n}^{\mathrm{DPP}}=R A_{n}^{L}-R A_{n}^{S}=A_{n}^{\mathrm{DPP}} \otimes h(k) \\
R B_{n}^{\mathrm{DPP}}=R B_{n}^{L}-R B_{n}^{S}=B_{n}^{\mathrm{DPP}} \otimes h(k)
\end{array} \quad n=1,2,\right.
$$

where $\otimes$ denotes convolution, $h(k)$ is the fiber impulse response, and $A_{n}^{\mathrm{DPP}}$ and $B_{n}^{\mathrm{DPP}}$ stand for the differential Golay-coded sequences [see Fig. 1(c)], being equivalent to

$$
\left\{\begin{array}{l}
A_{n}^{\mathrm{DPP}}=A_{n}^{L}-A_{n}^{S} \\
B_{n}^{\mathrm{DPP}}=B_{n}^{L}-B_{n}^{S}
\end{array} \quad n=1,2 .\right.
$$

Note that the process is similar to standard unipolar Golay-coded BOTDA using a pulse width equal to $T_{L}-T_{S}$ [see Fig. 1(c)], except that the BGS is not broadened due to the use of pulses longer than the acoustic-wave response time. The decoding process can be written as

$$
\begin{aligned}
& {\left[\begin{array}{l}
\left(R A_{1}^{\mathrm{DPP}}-R A_{2}^{\mathrm{DPP}}\right) *\left(A_{1}^{\mathrm{DPP}}-A_{2}^{\mathrm{DPP}}\right) \\
+\left(R B_{1}^{\mathrm{DPP}}-R B_{2}^{\mathrm{DPP}}\right) *\left(B_{1}^{\mathrm{DPP}}-B_{2}^{\mathrm{DPP}}\right)
\end{array}\right] /\left[2\left(N_{p}^{L}-N_{p}^{S}\right) L\right]} \\
& =h(k) \otimes\left[\begin{array}{l}
\left(A_{1}^{\mathrm{DPP}}-A_{2}^{\mathrm{DPP}}\right) *\left(A_{1}^{\mathrm{DPP}}-A_{2}^{\mathrm{DPP}}\right) \\
+\left(B_{1}^{\mathrm{DPP}}-B_{2}^{\mathrm{DPP}}\right) *\left(B_{1}^{\mathrm{DPP}}-B_{2}^{\mathrm{DPP}}\right)
\end{array}\right] /\left[2\left(N_{p}^{L}-N_{p}^{S}\right) L\right] \\
& =h(k) \otimes f_{S-C}^{\mathrm{DPP}},
\end{aligned}
$$

where $*$ denotes cross-correlation, $N_{P}^{L}$ and $N_{P}^{S}$ are the number of sampled points within the pulse width $T_{L}$ and $T_{S}$, respectively, $L$ is the code length, and $f_{S_{-} C}^{\text {DPP }}$ is the equivalent interrogating function, which possesses a triangular temporal shape having a width equal to $T_{L}-T_{S}$, as illustrated in Fig. 2 (see the red curve). It must be mentioned that although a sharp spatial resolution can be achieved by such a proper post-processing, eight Golay sequences still have to be sequentially launched into the sensing fiber (four with long pulses and four with short pulses), resulting in a time-consuming process.

Here we propose a novel hybrid Golay coding approach to realize DPP-BOTDA sensing, using only four code sequences, as shown in Fig. 1(d) which is designed as

$$
\left\{\begin{array} { l } 
{ A _ { 1 } ^ { h } = A _ { 1 } ^ { L } + A _ { 2 } ^ { S } } \\
{ A _ { 2 } ^ { h } = A _ { 2 } ^ { L } + A _ { 1 } ^ { S } }
\end{array} \text { and } \left\{\begin{array}{l}
B_{1}^{h}=B_{1}^{L}+B_{2}^{S} \\
B_{2}^{h}=B_{2}^{L}+B_{1}^{S}
\end{array} .\right.\right.
$$

This new coding structure takes advantage of the inherent features of Golay coding, namely the subtraction of complementary sequences, to directly obtain the following differential response:

$$
\begin{aligned}
& {\left[\begin{array}{c}
\left(R A_{1}^{h}-R A_{2}^{h}\right) *\left(A_{1}^{h}-A_{2}^{h}\right) \\
+\left(R B_{1}^{h}-R B_{2}^{h}\right) *\left(B_{1}^{h}-B_{2}^{h}\right)
\end{array}\right] /\left[2\left(N_{p}^{L}-N_{p}^{S}\right) L\right]} \\
& =h(k) \otimes\left[\begin{array}{l}
\left(A_{1}^{h}-A_{2}^{h}\right) *\left(A_{1}^{h}-A_{2}^{h}\right) \\
+\left(B_{1}^{h}-B_{2}^{h}\right) *\left(B_{1}^{h}-B_{2}^{h}\right)
\end{array}\right] /\left[2\left(N_{p}^{L}-N_{p}^{S}\right) L\right] \\
& =h(k) \otimes f_{\text {hybrid }}^{\mathrm{DPP}} \text {, }
\end{aligned}
$$

where $f_{\text {hybrid }}^{\text {DPP }}$ exhibits the same profile as $f_{S-C}^{\text {DPP }}$ in Fig. 2, featuring a spatial resolution determined by $T_{L}-T_{S}$. In addition to halving the measurement time, the proposed hybrid code can also improve the SNR of the decoded traces by $1.5 \mathrm{~dB}$ 
compared to the $\mathrm{S}-\mathrm{C}$ method, since the same response is obtained from half the number of traces, thus reducing the noise by a factor $\sqrt{2}$. Considering similar acquisition times, this leads to a $3 \mathrm{~dB}$ overall SNR enhancement over traditional unipolar Golay codes. Moreover, as each code symbol is non-zero in all the coding sequences, pulse power non-uniformity [13] is mitigated, securing the use of Golay codes without decoding distortion.

The proposed hybrid Golay-DPP is validated using the setup shown in Fig. 3, which is also used to evaluate the spatial resolution resulting from classical Golay-coded DPP-BOTDA based on S-C and C-S decoding. The light from a distributed feedback laser (DFB1) at $1549.6 \mathrm{~nm}$ is split by a 90:10 optical coupler. In the upper (probe) branch, an intensity modulator (IM1) biased at $V \pi$ is used to obtain a carrier-suppressed dualsideband (CS-DSB) probe wave. The IM1 is driven by a radiofrequency $(\mathrm{RF})$ signal generated from a microwave generator, which is used to scan the pump-probe frequency offset by a precise tuning of the RF signal frequency. A polarization scrambler is used to mitigate polarization fading on the traces. The dual-sideband probe signal is launched into a $10.164 \mathrm{~km}$ long sensing fiber through an optical isolator.

In the lower branch, Golay-coded optical pulse sequences are obtained using an intensity modulator (IM2) driven by a 512-bit electrical pulse sequence (61.44 $\mu$ s duration and $300 \mu$ s period), generated by an arbitrary waveform generator, corresponding to a coding gain of $10.5 \mathrm{~dB}$. In addition, a $61.54 \mu$ s optical pulse with a period of $300 \mu \mathrm{s}$ is applied to gate the coded optical pulse sequence, securing a high extinction ratio of the optical signal at the output of IM3. In order to avoid distortions caused by the slow transients of the EDFA, another optical pulse from DFB2 at a different wavelength $(1552 \mathrm{~nm})$ and a width of $1 \mu \mathrm{s}$ is launched into the EDFA1 slightly before the optical Golay sequences [14]. The $1552 \mathrm{~nm}$ optical beam is further filtered out through an optical bandpass filter. The flat amplified Golay code sequences are then launched into the sensing fiber through a TOA and an optical circulator. At the detection stage, the lower-frequency probe sideband is selected (i.e., to measure the Brillouin gain) by a fiber Bragg grating with a $3 \mathrm{~dB}$ linewidth of $6 \mathrm{GHz}$ after being amplified by EDFA2. The selected sideband is then sent into a $350 \mathrm{MHz}$ photodetector (PD) connected to an electrical oscilloscope for data acquisition.

The width of the long and short pulses are set to 30 and $25 \mathrm{~ns}$, corresponding to spatial resolutions of 3 and $2.5 \mathrm{~m}$, respectively, and a differential spatial resolution of $50 \mathrm{~cm}$. After propagating through the sensing fiber, three sets of optical Golay-coded pulse sequences are detected by the PD, corresponding to the sequences

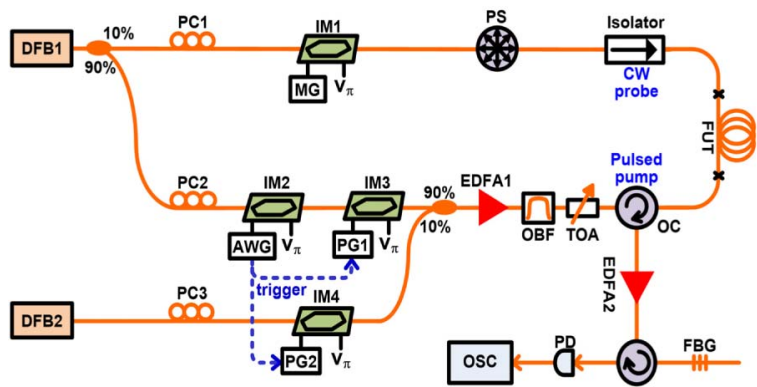

Fig. 3. Experimental setup. PG, pulse generator; OBF, optical bandpass filter; TOA, tunable optical attenuator.

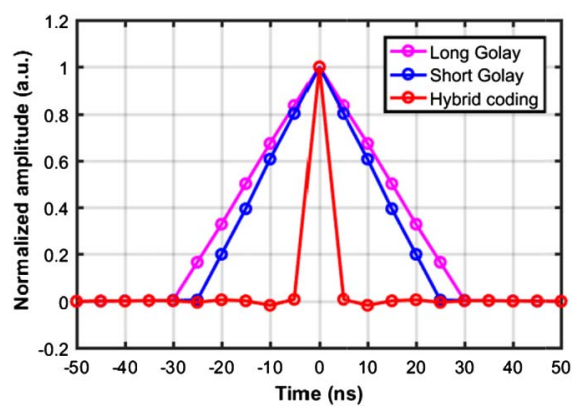

Fig. 4. Experimentally obtained Golay code interrogating functions for long-pulse, short-pulse and hybrid sequences.

with long pulses [Fig. 1(a)], with short pulses [Fig. 1(b)] and the hybrid DPP pulses [Fig. 1(d)]. The calculated FWHMs of crosscorrelation functions attributed to long Golay sequences, short Golay sequences, and hybrid code sequences (see Fig. 4) are $3,2.5$, and $0.5 \mathrm{~m}$, respectively, matching well the simulation results shown in Fig. 2.

Coded BOTDA traces at different scanning frequencies (from 10.7 to $10.9 \mathrm{GHz}$ with steps of $2 \mathrm{MHz}$ ) are acquired with 256 averages. After log normalization and decoding [13], a full map of the BGS is obtained along the sensing fiber. The BGSs measured at $1 \mathrm{~km}$ distance using long-pulse and short-pulse Golay sequences, and retrieved after decoding with a C-S method, S-C method, and the hybrid code are shown in Fig. 5. The results indicate respective linewidths of 51.0, 57.1, 33.3, 32.9, and $35.2 \mathrm{MHz}$, demonstrating that the C-S method, S-C method, and hybrid coding method do not broaden the BGS, as expected when using a DPP-BOTDA system [15].

Although both C-S and S-C approaches provide practically the same BGS width, different spatial resolutions are obtained as previously analyzed. To verify this, a hotspot is created near the far fiber end by placing $\sim 10 \mathrm{~m}$ of fiber in a water bath at a temperature of $50^{\circ} \mathrm{C}$, while the rest of the sensing fiber is at room temperature $\left(25^{\circ} \mathrm{C}\right)$. The $\mathrm{BFS}$ profiles, around the hotspot, processed using the two traditional Golay schemes (i.e., the S-C and C-S methods) are shown in Fig. 6. As expected, the $\mathrm{C}-\mathrm{S}$ method leads to a spatial resolution equivalent to the ones obtained with the long (non-differential) pulses. Using the same experimental dataset, Golay traces are then decoded by the $\mathrm{S}-\mathrm{C}$ method, and the retrieved BFS profile is shown in Fig. 6(b), where the BFS profile obtained with the C-S method is also presented for comparison. The results clearly indicate

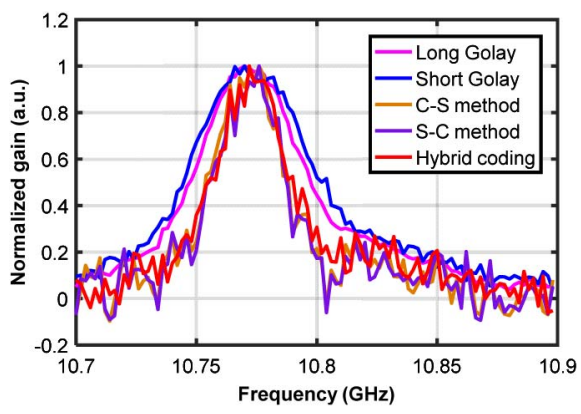

Fig. 5. Measured BGS at $1 \mathrm{~km}$ distance using long-pulse and shortpulse sequences and after decoding using an S-C method, C-S method, and the proposed hybrid coding scheme. 

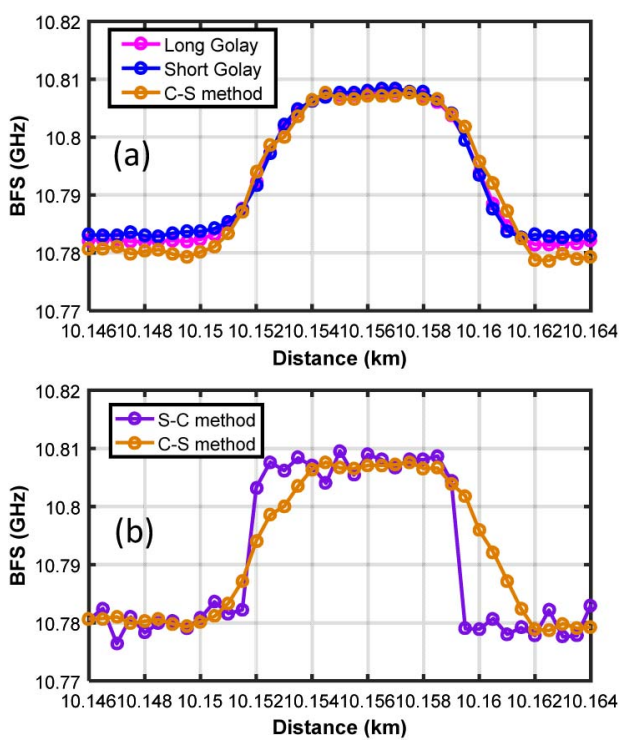

Fig. 6. (a) BFS around hotspot when using long-pulse Golay sequences, short-pulse Golay sequences, and DPP-pulses resulting after decoding with the C-S method. (b) Comparison of the BFS profile around hotspot obtained when using classical DPP-BOTDA with C-S and S-C decoding.
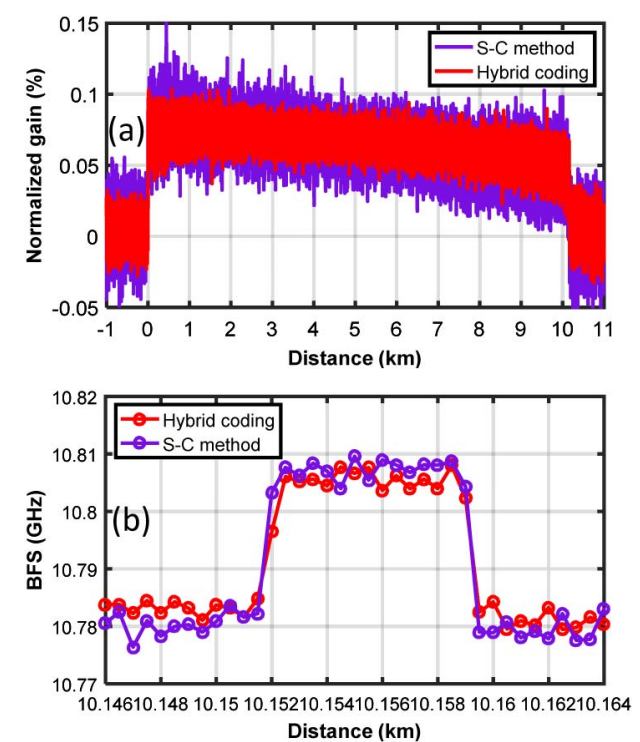

Fig. 7. (a) Decoded Brillouin gain traces at $10.78 \mathrm{GHz}$. (b) BFS around the hotspot section.

that a sharper BFS transition is obtained by the S-C method (corresponding to a $50 \mathrm{~cm}$ spatial resolution), demonstrating that the only way to achieve the DPP spatial resolution when using conventional Golay codes is to use the S-C approach.

The experiment is then repeated with the same experimental conditions, but using the proposed hybrid Golay sequences
[Fig. 1(d)]. The decoded Brillouin gain traces at the resonance frequency are shown in Fig. 7(a), for the proposed hybrid Golay scheme and the S-C method (used as reference). An SNR difference of about $1.5 \mathrm{~dB}$ can be observed when comparing the two traces, which is in good agreement with the expected behavior. The retrieved BFS profiles around the hotspot are shown in Fig. 7(b), for the same two cases, proving the high spatial resolution of $50 \mathrm{~cm}$ achieved by the hybrid DPP-Golay code (fully similar to the S-C approach). The BFS uncertainty is estimated to be $1.4 \mathrm{MHz}$ by calculating its standard deviation from 10.14 to $10.148 \mathrm{~km}$.

In conclusion, a theoretical and experimental analysis of Golay coding in DPP-BOTDA has been reported, investigating different approaches for coding/decoding. In particular, a novel hybrid coding method combining the features of unipolar Golay coding to get a response equivalent to differential pulses has been proposed and experimentally validated, verifying that the hybrid coding method can secure sharp (sub-meter) spatial resolution, while providing an overall $3 \mathrm{~dB}$ SNR enhancement when compared to a conventional Golay coding implementation (considering the same measurement time). Although the higher-order pump depletion compromises the overall performance [13], the presented concept can be implemented with bipolar Golay sequences $[9,10]$ to further improve the performance of coded DPP-BOTDA sensors.

Funding. Swiss Commission for Technology and Innovation (18337.2 PFNM-NM); China Scholarship Council (CSC); National Natural Science Foundation of China (NSFC) (61735015).

\section{REFERENCES}

1. T. Horiguchi, K. Shimizu, T. Kurashima, M. Tateda, and Y. Koyamada, J. Lightwave Technol. 13, 1296 (1995).

2. M. A. Soto and L. Thévenaz, Opt. Express 21, 31347 (2013).

3. M. Alem, M. A. Soto, M. Tur, and L. Thévenaz, Proc. SPIE 10323, 103239J (2017).

4. W. Li, X. Bao, Y. Li, and L. Chen, Opt. Express 16, 21616 (2008).

5. S. M. Foaleng, M. Tur, J.-C. Beugnot, and L. Thévenaz, J. Lightwave Technol. 28, 2993 (2010).

6. M. Alem, M. A. Soto, and L. Thévenaz, Opt. Express 23, 29514 (2015).

7. A. Domínguez-López, Z. Yang, M. A. Soto, X. Angulo-Vinuesa, S. Martín-López, L. Thévenaz, and M. González-Herráez, Opt. Express 24, 10188 (2016).

8. M. A. Soto, G. Bolognini, F. Di Pasquale, and L. Thévenaz, Opt. Lett. 35, 259 (2010).

9. M. A. Soto, S. Le Floch, and L. Thévenaz, Opt. Express 21, 16390 (2013).

10. Z. Yang, M. A. Soto, and L. Thévenaz, Opt. Express 24, 586 (2016).

11. M. A. Soto, M. Taki, G. Bolognini, and F. Di Pasquale, Opt. Express 20, 6860 (2012).

12. M. Nazarathy, S. A. Newton, R. P. Giffard, D. S. Moberly, F. Sischka, W. R. Trutna, and S. Foster, J. Lightwave Technol. 7, 24 (1989).

13. Z. Yang, Z. Li, S. Zaslawski, L. Thévenaz, and M. A. Soto, Opt. Express 26, 16505 (2018).

14. F. Wang, C. Zhu, C. Cao, and X. Zhang, Opt. Express 25, 3504 (2017).

15. A. Minardo, R. Bernini, and L. Zeni, Opt. Express 19, 19233 (2011). 